\title{
CONCEPTUAL DESIGN FOR ASSEMBLY IN AEROSPACE INDUSTRY: SENSITIVITY ANALYSIS OF MATHEMATICAL FRAMEWORK AND DESIGN PARAMETERS
}

\author{
Formentini, Giovanni (1); \\ Favi, Claudio (1); \\ Cuiller, Claude (2); \\ Dereux, Pierre-Eric (2); \\ Bouissiere, Francois (2); \\ Jurbert, Cédric (2) \\ 1: University of Parma; \\ 2: Airbus S.A.S.
}

\begin{abstract}
One of the most challenging activity in the engineering design process is the definition of a framework (model and parameters) for the characterization of specific processes such as installation and assembly. Aircraft system architectures are complex structures used to understand relation among elements (modules) inside an aircraft and its evaluation is one of the first activity since the conceptual design. The assessment of aircraft architectures, from the assembly perspective, requires parameter identification as well as the definition of the overall analysis framework (i.e., mathematical models, equations).

The paper aims at the analysis of a mathematical framework (structure, equations and parameters) developed to assess the fit for assembly performances of aircraft system architectures by the mean of sensitivity analysis (One-Factor-At-Time method). The sensitivity analysis was performed on a complex engineering framework, i.e. the Conceptual Design for Assembly (CDfA) methodology, which is characterized by level, domains and attributes (parameters). A commercial aircraft cabin system was used as a case study to understand the use of different mathematical operators as well as the way to cluster attributes.
\end{abstract}

Keywords: Product architecture, Product modelling / models, Conceptual design, Aicraft systems, Design for Assembly (DFA)

\section{Contact:}

Formentini, Giovanni

University of Parma

DIA (Engineering and Architecture Department)

Italy

giovanni.formentini@unipr.it

Cite this article: Formentini, G., Favi, C., Cuiller, C., Dereux, P.-E., Bouissiere, F., Jurbert, C. (2021) 'Conceptual

Design for Assembly in Aerospace Industry: Sensitivity Analysis of Mathematical Framework and Design Parameters', in Proceedings of the International Conference on Engineering Design (ICED21), Gothenburg, Sweden, 16-20 August 2021. DOI: $10.1017 /$ pds.2021.73 


\section{INTRODUCTION}

The development of a new product (product development process - PDP) is a complex activity where several engineering disciplines are involved. The PDP is part of the engineering design process and it consists of few phases: i) conceptual design, ii) embodiment design, and iii) detail design (Pahl and Beitz, 2007). To support and optimize the PDP different methods and tools have been developed. These methods are called Design for X (DfX), where the $\mathrm{X}$ is substituted with the optimization goal. The earliest DfX methods developed were focused on the optimization of the product manufacturing and assembly aspects (Krishnan \& Ulrich, 2001; Selvaraj et al., 2009; Favi et al., 2016). It has been shown that the assembly phase might impact up to the $40 \%$ of the final product cost (Pahl and Beitz, 2007) and this savings is more relevant for large and complex products, such as an aircraft (Bullen, 1999). Design for assembly methods are well-know and largely applied in industries with good results: the BoothroydDewhurst (B\&D) (Boothroyd et al., 2011), the Hitachi method (AREM) (Suzuki et al., 2003), and the Lucas method (Stone et al., 2004). Unfortunately, these methods require information of high granularity, only available at late design phase. To avoid this drawback, it is possible to anticipate the application of these methods at earlier design phases (i.e., conceptual phase). The main challenge working at the conceptual phase is the quantity and the type of information available. Indeed, information is characterized by low level of granularity and usually represented using functional schemes (Favi et al., 2018). Functional schemes are valuable tools especially for creating modular products, where a module is defined as a group of functional carriers, meaning a component or element that clusters different functions together (Baylis et al., 2018, Bonvoisin et al., 2016). Many principles are available in literature to obtain modular products (Yu et al., 2011; AlGeddawy and ElMaraghy 2013) and an interesting overview of methods available in literature is proposed by Bonvoisin et al. (2016). The description of how functions and modules are allocated to physical components is called product architecture (Chen et al., 2018). Some authors tried to anticipate the application of DFA methods at the conceptual design phase, working with product architectures (Stone et al., 2004; Favi and Germani, 2012). A method called "Conceptual Design for Assembly" (CDfA) was proposed by Bouissiere et al. (2019) with the aim to assess assembly complexities in aircraft industry, analysing product architecture concepts at the early design phase. Although this method seems to provide results which are fitting with main issues observed in the assembly line, it is not clear the implication that each parameter of the proposed method has on the result. To address the influence of design parameters on a given model, sensitivity analysis (SA) is considered a suitable tool. SA is a method for quantifying parameters uncertainty in any type of model (Marino et al., 2008; Ravalico et al., 2005; Hamby, 1994) by two different approaches: i) local analysis and ii) global analysis. The former focuses on one parameter at time, assessing its variation with respect to its nominal value; the latter tries to understand how the change of multiple parameters may affect the model result (Campolongo et al., 2007). One of the most used method used for local analysis is the OneFactor-At-Time (OFAT) that consists of changing one parameter at times, keeping other fixed (Saltelli et al., 2006). The OFAT approach is well-known in literature and it has been used in many fields (i.e., environmental, financial, etc.) (Saltelli and Annoni, 2010). However, many authors state OFAT method is not reliable to identify parameters correlation, especially in complex models such as environmental models, climate models, etc. (Czitrom, 1999; Saltelli and Annoni, 2010; Pianosi et al., 2016).

The aim of this paper is to propose a SA for the CDfA methodology proposed by Bouissiere et al. (2019), to understand how the results of the CDfA model developed to describe the system of interest is affected by the model itself (mathematical operators) and parameters (herein called attributes). The framework behind the CDfA methodology is characterized by domains (which are grouping different attributes belonging to a common aspect), levels (which are decomposing the original problems in discrete sub-problems based on system invariants) and mathematical equations that are used to combine attributes inside domains and between levels. The paper investigates the meaning of using different mathematical operator for clustering attribute's scores inside domain by analysing three operators: (i) the Root Mean Square (RMS), (ii) the Mean, and (iii) a custom operator developed for the system of interest. The method used in this paper is the One-Factor-At-Time (OFAT) due to: (i) the easiness of the mathematical model analysed, (ii) the assumption of model linearity, and (iii) the origin of data (i.e., the model analysed was not obtained through experiments). RMS is chosen every time a conservative result is desired and a large discrepancy among scores is noticed (i.e., to cluster scores of attributes inside the same domain due to their variability) while the Mean operator is chosen when score's attributes has been already processed (i.e., to cluster scores of different domains). The 
novelty of the paper lies in the possibility to use the results obtained by the SA to help designers and product architects to accurately understand the CDfA model used and to tune it accordingly to the system of interest. The tuning process can be performed in several ways: (i) by changing the number of attributes inside domains, (ii) by changing domains level, and (iii) by modifying the model equations (i.e., changing the mathematical operator or using a specific weight). However, the weighing process is not recommended since it will introduce engineer judgments inside the model. A specific case study concerning the application of the CDfA methodology to the aircraft cabin equipping is reported within this study to show how the model and the related parameters fit with the criticalities observed in the cabin assembly line. The outcome allows to understand the implications of attributes, domains, and levels on the overall final score. After this Introduction (Section 1), the method used to perform the sensitivity analysis is described in Section 2. Then, the cabin of a commercial aircraft is analysed as a relevant case study (Section 3). Results are discussed in Section 4 and, finally, Conclusions are presented in Section 5.

\section{MATERIAL AND METHOD}

The Conceptual Design for Assembly (CDfA) is a method aiming at assessing assembly complexities of product architectures (i.e., module installation) at the conceptual design phase (Bouissiere et al. (2019)). The method consists of a framework developed by the definition of three types of information: (i) attributes, (ii) domains and (iii) levels. An attribute (A) is a key feature that influences assembly operations, and it is referred to a specific aspect related to the assembly operation. A domain is a cluster of one or more attributes that address the same assembly aspect and provides the same meaning for each designer/engineer. Finally, a level is defined as a group of domains. Levels are used to divide the main problem (i.e., overall product architecture) into sub-groups of smaller complexities. To move from one level to another one is necessary to define a product invariant. A product invariant is a design feature that does not change and cannot be changed withing the product under study. Once the model is completed, a hierarchical structure is obtained (Figure 1).

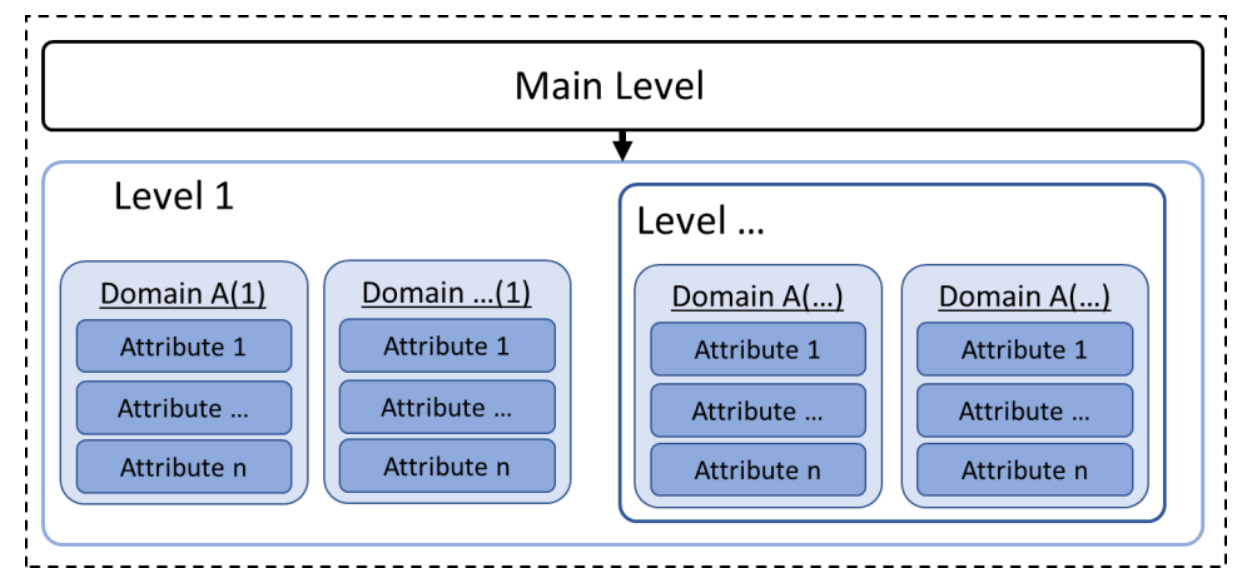

Figure 1. CDfA hierarchical structure

Attributes represent different heterogeneous information, and they need to be normalized before to combine them in a given model. Thus, using scoring matrices it is possible to translate attribute data (numbers or strings) in score ranging from one (1) to five (5) (Favi et al., 2020). Finally, after the normalization phase, scores are aggregated by mathematical operators (equations). Starting from the lowest level, attributes' scores inside a domain are collected using a function $g(\cdot)$ to obtain one single score per domain for each analysed element (i.e., module). The process is repeated for all domains inside the level. Then, domains' scores are moved to the upper level using a function $f(\cdot)$. The function $f(\cdot)$ is chosen based on the level invariant. The overall process (i.e., collection of attributes' score with function $g(\cdot)$ and collection of domains' score with function $f(\cdot)$ ) is repeated until the Main Level is reached. The Main Level assembles all domains defined in the hierarchical structure with a single score for each module analysed within the system of interest. To keep the model straightforward, the process of aggregation is performed using simple mathematical operators such as the Mean operator, the Root Means Square, the Maximum operator, the Minimum operator, etc. Then, when results are obtained, it is possible to tune the model by using different operators with the aim to better resemble the reality of the 
system under analysis. Further explanations regarding the CDfA approach and the modelling process can be found in works of the same authors (Formentini et al., 2020; Favi et al., 2020; Bouissiere et al., 2019). Since the mathematical model of the CDfA is done independently from the definition of attributes, domains, and levels, it requires an impact assessment on the final score. The sensitivity analysis is a handful tool that allows to clear understand the correlation among parameters on a model. Among all the techniques available in literature, the One-Factor-At-Time (OFAT) approach is used to perform the sensitivity analysis on the CDfA hierarchical structure. The OFAT approach consist of: (i) defining a baseline for the parameters under study, (ii) varying one parameter at time, keeping other fixed, (iii) computing the variation of input with respect to the out, and (iv) computing the sensitivity as the ratio between $\Delta$ output and $\Delta$ input. An attribute is considered more sensitive with respect to the output if a slightly change of the attribute value will generate a significant output change. From a geometrical point of view, the sensitivity of a parameter is the angle $\alpha$ (Figure 2).

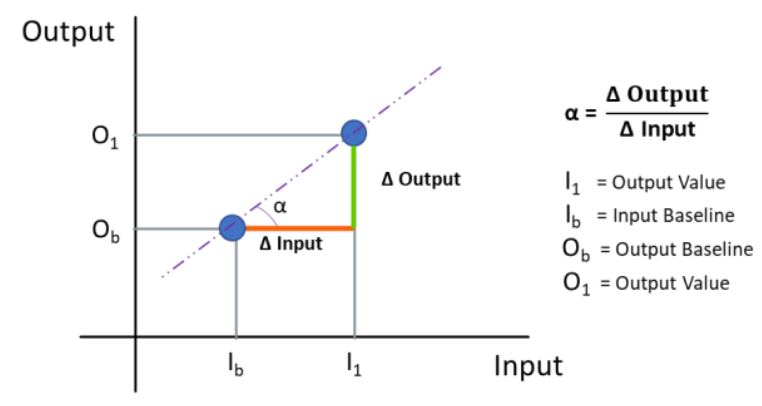

Figure 2. Attribute sensitivity geometrical interpretation

The sensitivity analysis on the CDfA methodology is performed following 4 steps (Figure 3).

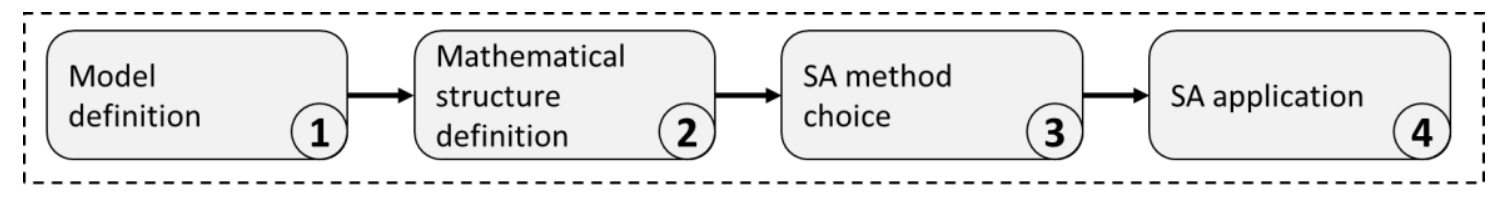

Figure 3. SA workflow

The first step (Model definition) consists of creating the CDfA framework (model structure) that will be used to model the architecture of the product under study. Indeed, the CDfA framework is a general structure that can be applied for all aircraft's systems to assess manufacturing and assembly aspects, but it needs to be specialized according to the chosen system of interest (i.e., nose-fuselage, cabin, etc.). Thus, different CDfA frameworks might be obtained according to the product considered, to the information available and to elements analysed (i.e., modules or interfaces). Once the framework is obtained, the second step (Mathematical structure definition) consists of choosing mathematical operators to link scores inside the defined hierarchical structure. Along with the mathematical operators used, different parameters sensitivity may be achieved. It is possible to perform the SA multiple times changing mathematical operators to understand which mathematical operator is more suitable for the desired application. The third step (SA method choice) consist of determining the sensitivity analysis method. The choice of the SA is strictly related to the mathematical model defined in second step. For instance, if equations chosen to collect scores present attributes interdependencies a global SA method should be preferred. The final step deals with the assessment of the attribute's sensitivity (SA application). The analysis can be performed using already available tools or, according to the complexity of the SA method chosen, it is possible to automatize the SA using spreadsheets.

\section{CASE STUdY}

The OFAT SA was performed on the CDfA model created to assess the assembly complexity of Cabin modules of the aircraft Airbus A330 (Figure 4). 


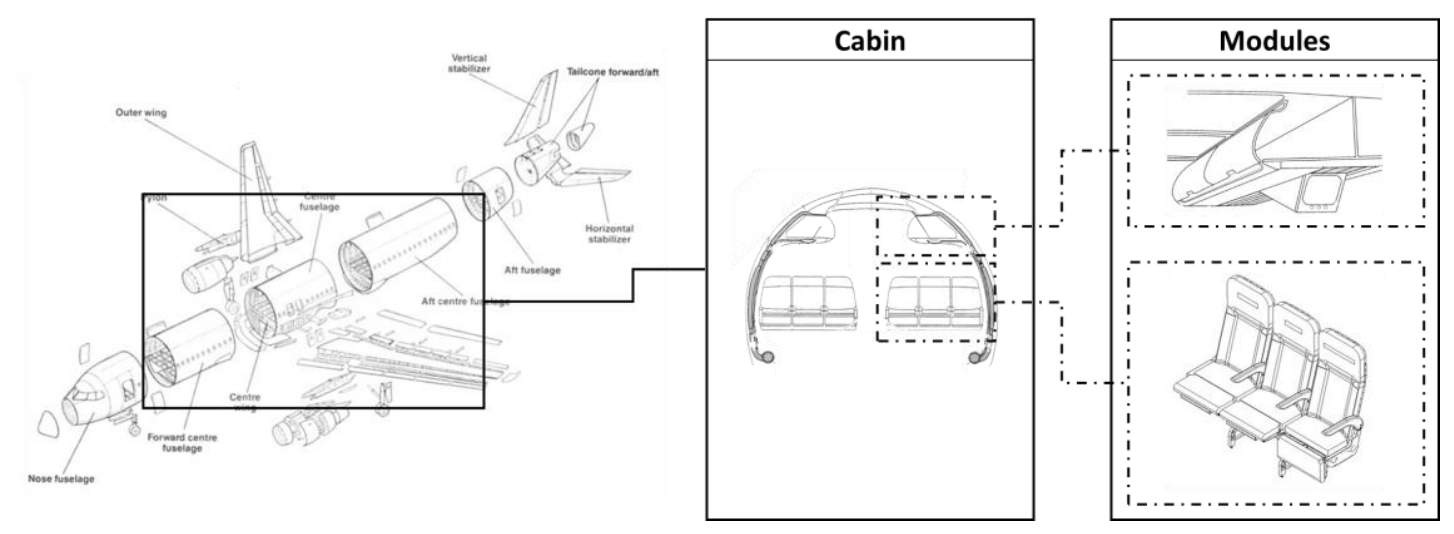

Figure 4. A330 cabin and modules

The model created for the cabin assessment is composed by only one level due to the characteristic of the system of interest (cabin) that does not require a further discretization in additional levels. In the first level, four different domains were identified: i) mechanical domain with five attributes, ii) furnishing domain with two attributes, iii) system interface domain with five attributes, and iv) handling domain with four attributes. The hierarchical structure is represented in Figure 5, where attributes are indicated with their ID (e.g., A.1, A.2, A.3, etc.) and their name. For the sake of brevity, in the following paragraph, attributes are identified only by their ID.

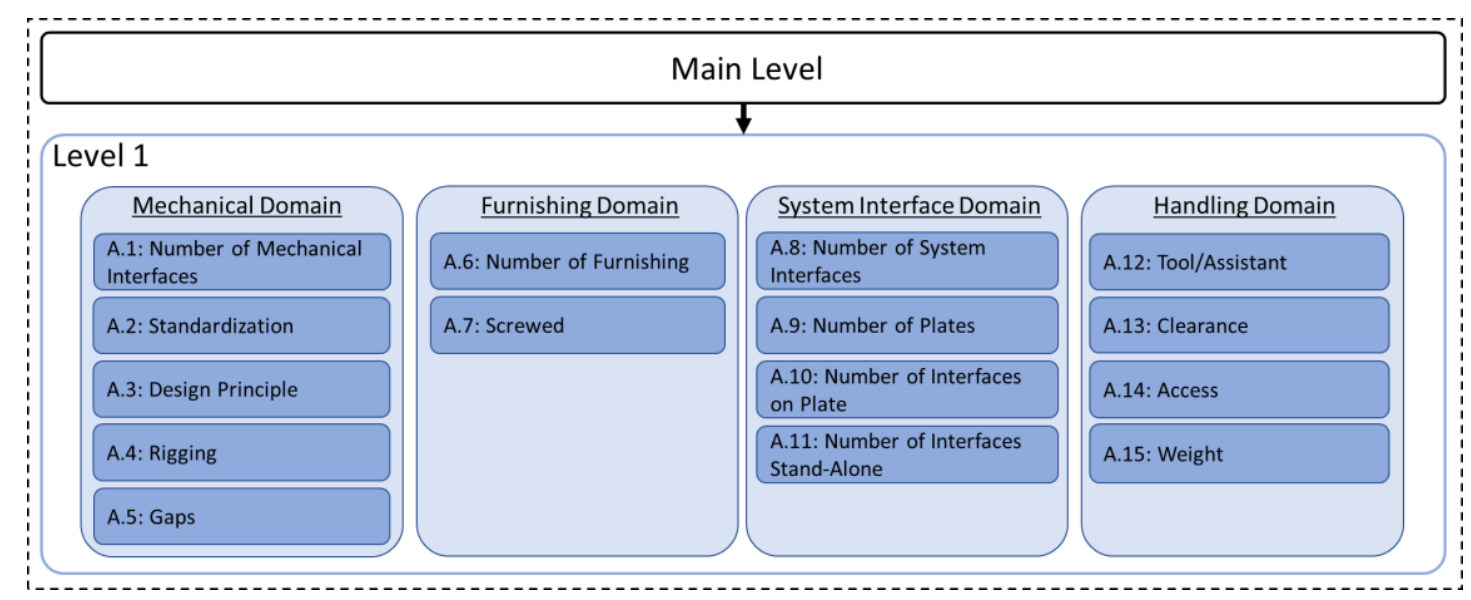

Figure 5 - Cabin hierarchical structure

The analysis was performed to assess the assembly complexity of modules inside the cabin. A module is an element that by means of tools need to be fixed inside the cabin and linked with mechanical and system interfaces. For instance, hat racks, toilets, seats, and galley are considered cabin modules. According to the cabin analysed, different modules and different quantities might be present. The mechanical domain collects attributes referring to the mechanical fixation of modules to the aircraft structure, the furnishing domain clusters attributes expressing the modules' aesthetics features, while the system interface domain groups attributes reflecting the interfaces (electrical connections, pipes, etc.) that are required by modules to work properly and, finally, the handling domain collects attributes referring to the module handling process (i.e., move the module inside the cabin, keep the module in place while installing it, etc.). The analysis was performed using results of the CDfA for a given architecture and two modules were used as baseline:

- baseline 1 (BS1) - the module Hat-Rack A,

- baseline 2 (BS2) - the module Galley B.

The analysis was repeated two times (i.e., I, II) using different mathematical operators to cluster attributes inside domains. Specifically, for the first analysis (I), the mechanical, furnishing and handling domains were clustered with equation 1 (RMS), while the System Interface Domain with equation 3. For the second analysis (II) the mechanical, furnishing and handling domains were clustered with equation 2 (Mean) while the System Interface Domain with equation 4. Equations 3 and 4 used for the System Interface Domain were developed according to the engineering knowledge to better model the CDfA Cabin outcomes. 
(1) Mechanical/Furnishing/Handling Domain Score (I) $=\sqrt{\frac{\sum_{i=1}^{n}(A . i)^{2}}{n}}$

(2) Mechanical/Furnishing/Handling Domain Score ${ }_{(I I)}=\sum_{i=1}^{n} \frac{A . i}{n}$

(1) System Interface Domain score $(I)=\sqrt{\frac{\left(\left(1+4 *\left(\frac{A .10}{A .9+A .10}\right)\right)+\left(\frac{A .8}{A .9}\right)\right)^{2}+(A .11)^{2}}{2}}$

(2) System Interface Domain score $(I I)=\sqrt{\frac{\left(\left(1+4 *\left(\frac{A .10}{A .9+A .10}\right)\right)+\left(\frac{A .8}{A .9}\right)\right)^{2}+(A .11)^{2}}{2}}$

Finally, the domain scores obtained were collected with a Mean operator for each module that is installed within the cabin. The OFAT method was implemented as a macro inside a spreadsheet. For each baseline, one attribute at time was changed, keeping others fixed and the value $\Delta$ Input (equation 4), $\Delta$ Output (4 5) and Final Score were recorded for each result and the Sensitivity (equation 6) was computed.

(3) $\Delta$ Input $=\mid$ Baseline Input - Input Value $\mid$

(4) $\Delta$ Output $=\mid$ Baseline Final Score - Final Score $\mid$

(5) Sensitivity $=\frac{\Delta \text { Output }}{\Delta \text { Input }}$

For the sake of brevity, results obtained with BS2 (Galley B), for the attribute Number of Mechanical Interfaces during the two runs are presented in Figure 6. It is worth noting that, when the input value is equal to the benchmark value, the sensitivity cannot be computed (i.e., $N / D$ value is shown).

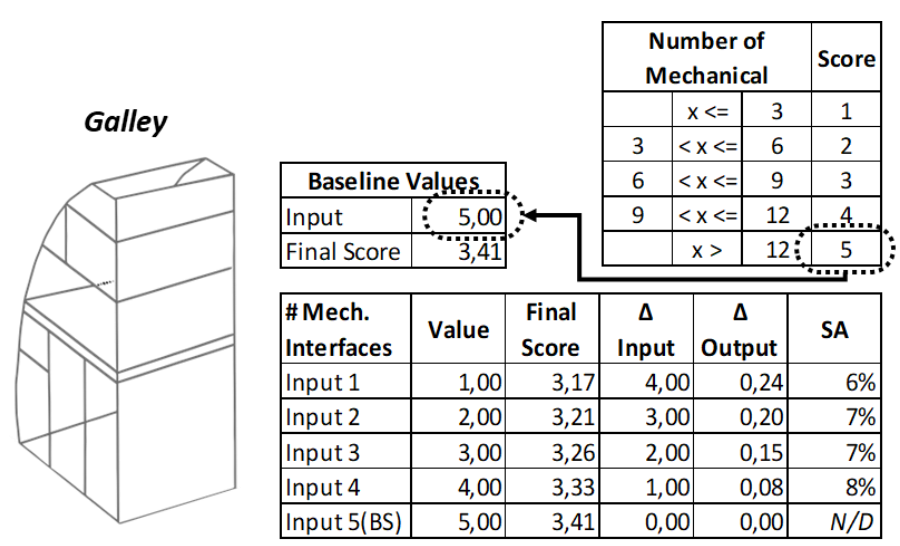

a)

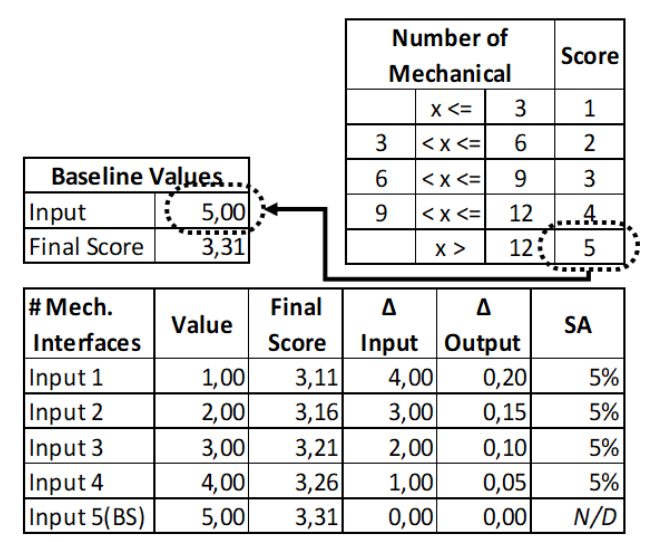

b)

Figure 6. Number of mechanical interfaces (BS2 - Galley B) a) run I, b) run II

\section{RESULTS AND DISCUSSIONS}

The first analysis was run to understands the sensitivity of the CDfA results when the RMS operator is used to collect data inside a domain. In the case of the cabin, $R M S$ operator is used for three of the four domains (mechanical, handling and furnishing). Here below are reported two results (Figure 7) concerning the two baselines (BS1 Hat Rack A (left) and BS2 - Galley B (right)), in the case of the mechanical domain where five attributes are aggregated by $R M S$ operator. The two graphs report in the $\mathrm{x}$-axis the input values (i.e., from 1 to 5 ) and in the $\mathrm{y}$-axis the related sensitivity values. It is worth noting that one attributes (i.e., A.1) has all the values within the range, while other attributes have only a limited set of values within the same range. This aspect is due to possible alternatives (i.e., input values) that a given attribute can adopt. When different baselines (i.e., modules) are considered, the general trend is confirmed, meaning the sensitivity of all attributes increases together with the input value. However, few differences may be highlighted: in the BS1, A.5 presents the highest sensitivity together with $A .1$ while for BS2, the A.5 sensitivity drops down to the lowest value together with A.2. 
The reason lies in the $\Delta$ input and $\Delta$ output values obtained by opposite input values ( 1 for BS1; 5 for BS2) and different CDfA final score values (2,32 for BS1; 3,68 for BS2). Moreover, the sensitivity varies for all attributes, according to the input value. For instance, looking at BS2, the sensitivity for A.1 is $6 \%$ when the input is 1 and increases to $8 \%$ when the input is 5 . This trend is shared by all attributes and it is justifiable by analysing the $R M S$ operator; in fact, it is a non-linear operator. Another interesting result is obtained by looking at the distribution of each attribute, even though all attributes present in the domain have the same range of input values (i.e., from 1 to 5) they have different sensitivity values. For instance, in the case of BS2 - Galley B, when the input is 2 , the A.5 has a sensitivity of approximately $2 \%$ while $A .4$ presents a sensitivity of approximately $4 \%$. The reason lies in the input baseline values which are different for the two attributes.
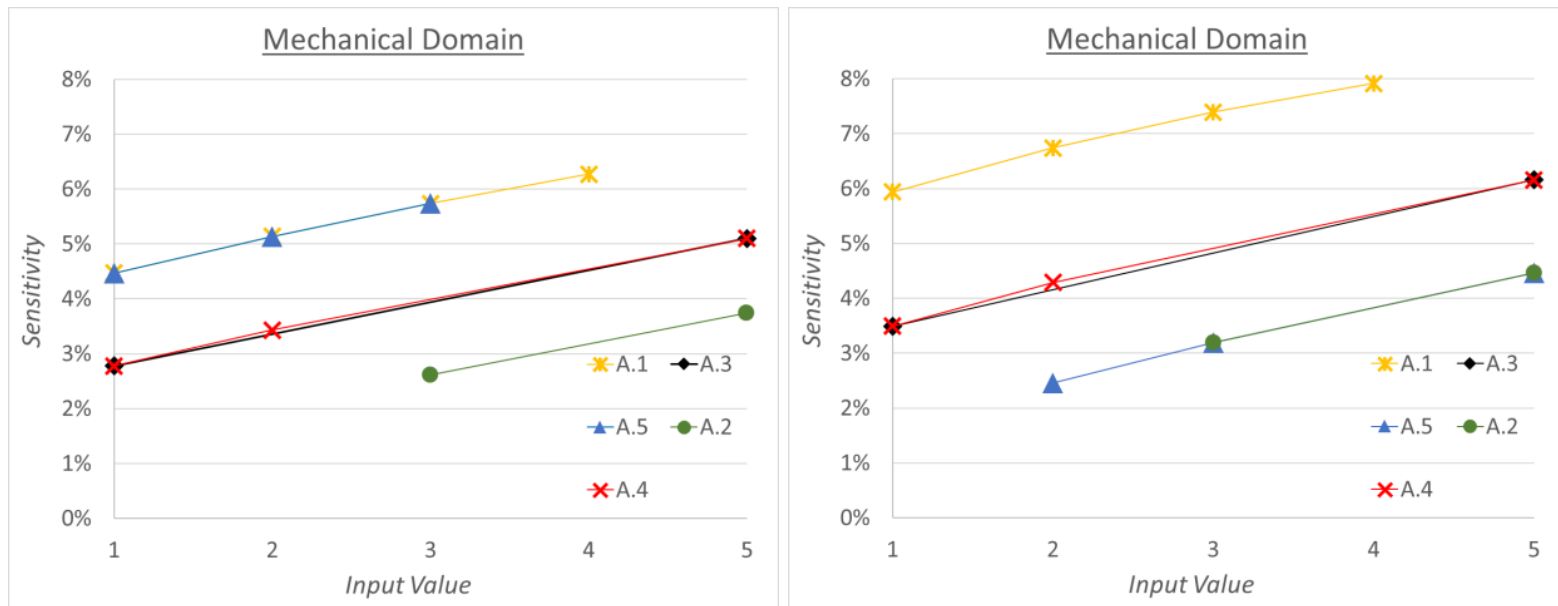

Figure 7. Sensitivity results of BS1 - Hat Rack A (left) and BS2 - Galley B (right); mechanical domain attributes; run I

Finally, plotting together the results for the mechanical domain and the furnishing domain (Figure 8) which used the same mathematical operator $(R M S)$ it can be observed that attributes (A.6 and A.7) within the second domain (furnishing domain) have in general higher sensitivity than the attributes (A.1, A.2, A.3, A.4 and A.5) within the first domain (mechanical domain). The reason lies in the number of attributes presents in each domain; indeed, the furnishing domain collects two attributes while the mechanical domain presents five attributes. Less attributes means that each of them has a greater impact on the overall domain score and then the final score. These two results lead to important consequence when the CDfA model is created: the more attributes are clustered together inside a domain, the less each attribute will contribute on the overall score. The same results are obtained repeating the analysis for the handling domain.

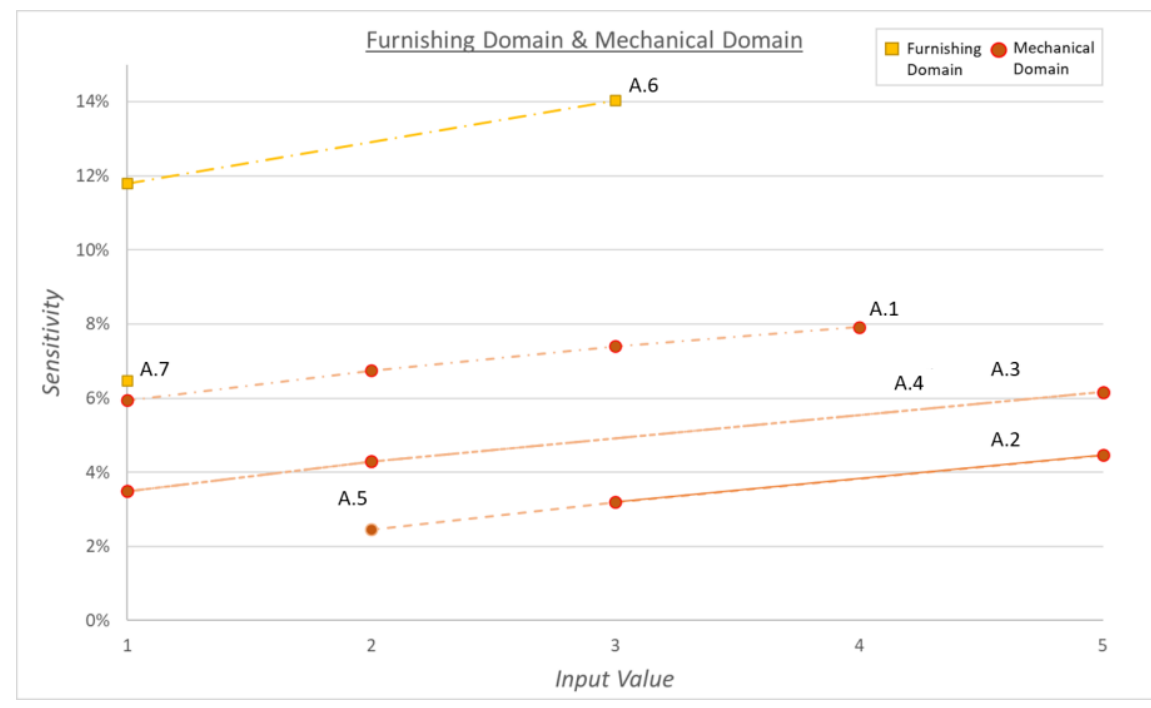

Figure 8. BS2 - Galley B; furnishing \& mechanical domain attributes; run I 
The second analysis was run to understands the sensitivity of the CDfA results when the Mean operator is used to collect data inside a domain. Again, within the specific case of the cabin, Mean operator replace $R M S$ operator for three of the four domains (mechanical, handling and furnishing). Results highlight that in case the Mean operator is chosen all attributes inside a domain have the same sensitivity value regardless the input values (i.e., baselines). In fact, the Mean operator is a linear operator and the sensitivity for an attribute (angle between Final Result and $\Delta$ Input) remains constant. In Figure 9 is shown the comparison of the sensitivity for mechanical domain attributes between the two baselines (BS1 Hat Rack A (left) and BS2 - Galley B (right)), when the Mean operator is used. Both analyses show a sensitivity of $5 \%$ regardless the baseline.
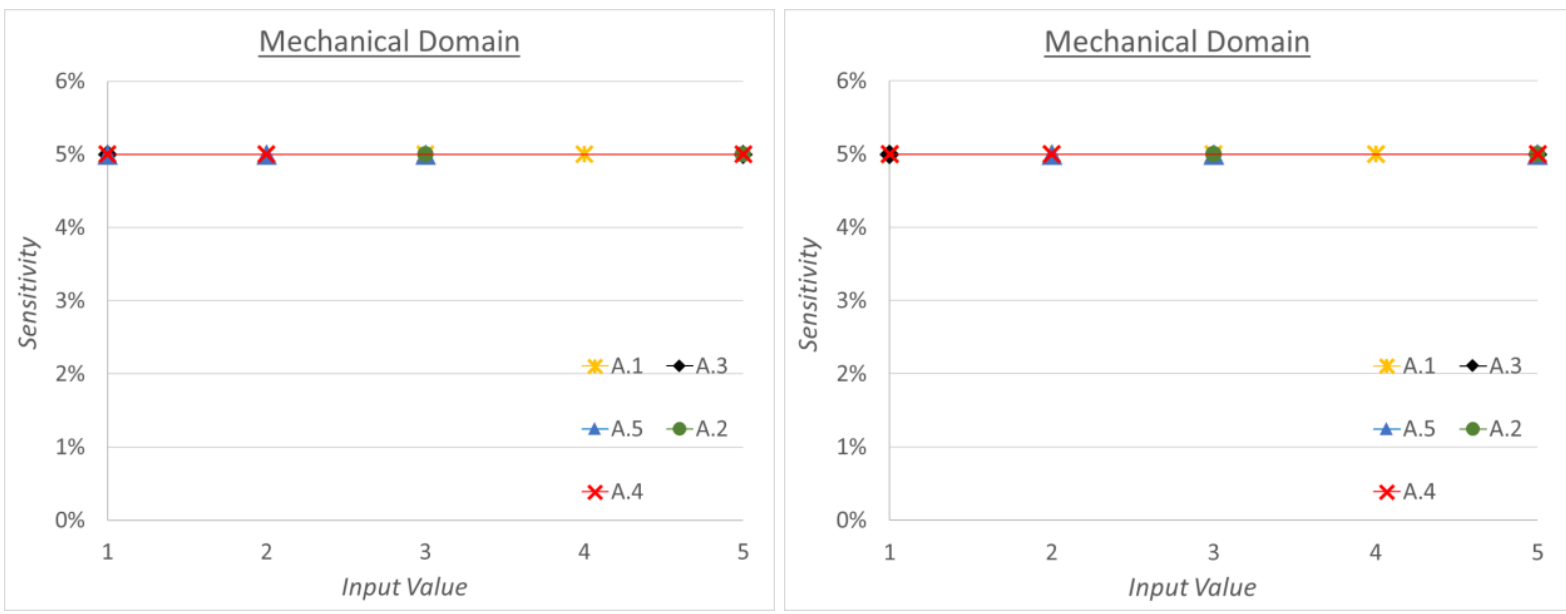

Figure 9. Sensitivity results of BS1 - Hat Rack A (left) and BS2 - Galley B (right); mechanical domain attributes; run II

As already shown for the RMS operator, when more attributes are collected inside a domain, the sensitivity of each attribute decrease. This trend is confirmed by the results plotted in Figure 10.

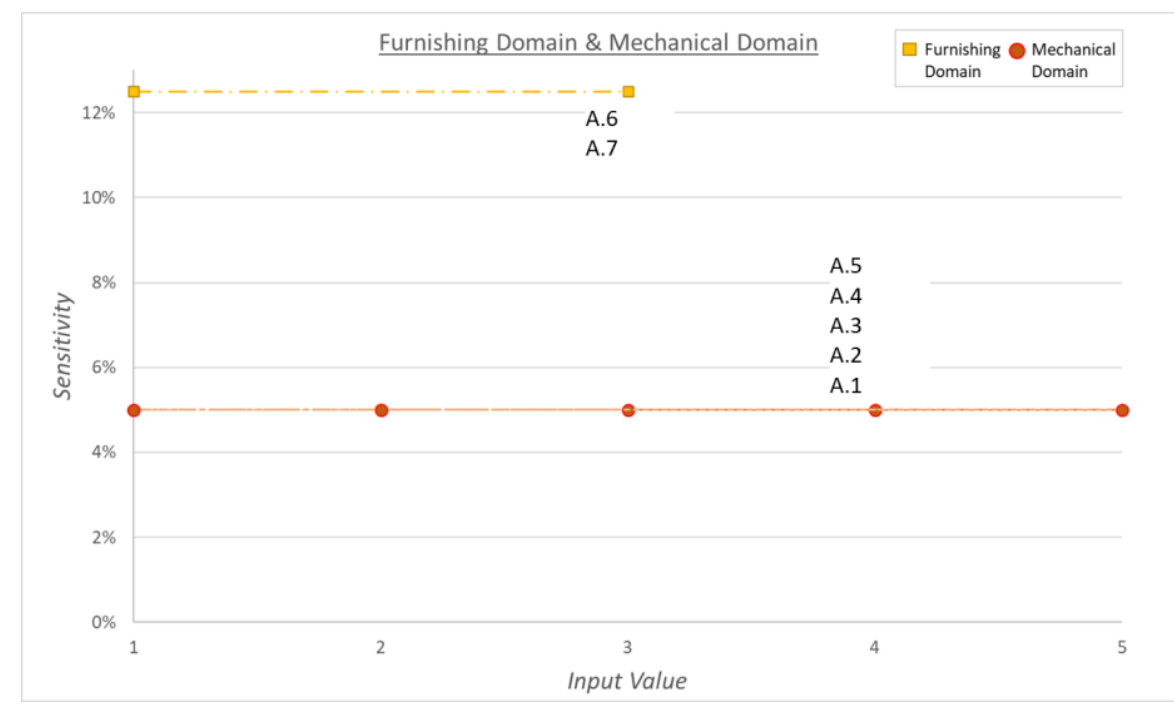

Figure 10. BS1; Mechanical \& furnishing domain attributes; run II

For the CDfA method applied to the Cabin case study, the system interface domain requires a separated analysis, due to the mathematical equation used to collect attributes inside this domain. In fact, by running the SA, results shown in Figure 11 are obtained. 


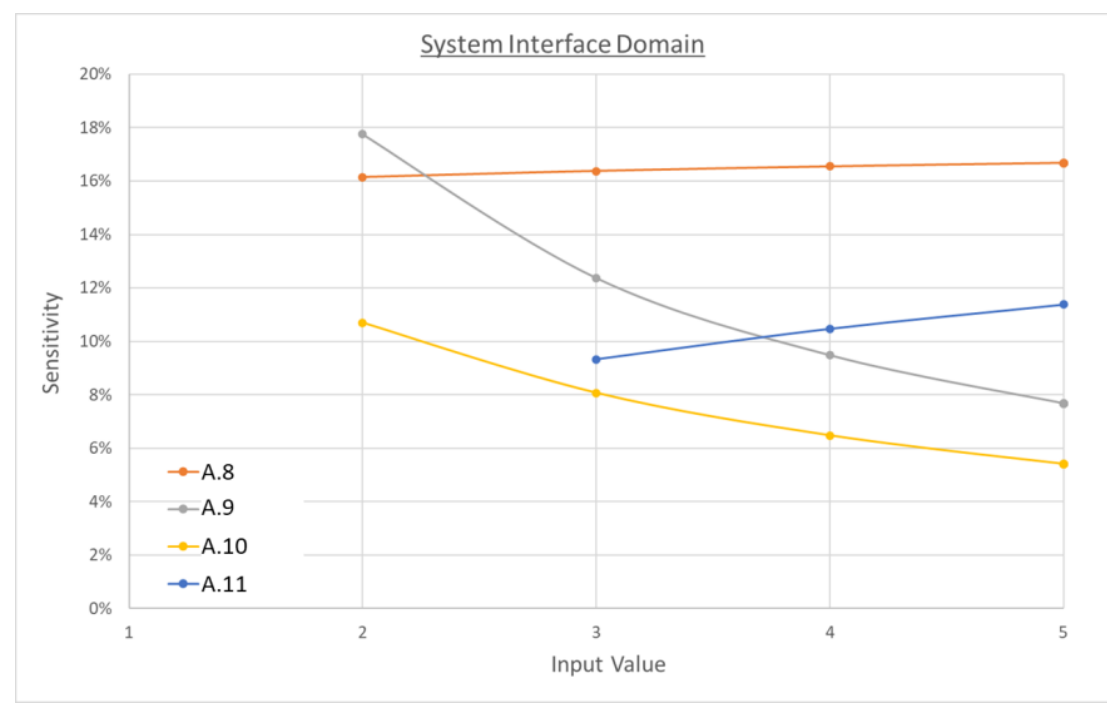

Figure 11. BS1; System interface domain attributes; run I, run II

The mathematical equation used to collect scores inside the system interface domain was obtained through engineering consideration. The sensitivity for each attribute shows a random result that does not resemble any specific trend. All attributes present different sensitivity value according to the input, in fact the used equation is not linear. Moreover, $A .8$ and $A .11$ increase their sensitivity with the input value indeed in equation (1) they are both at the numerator, while $A .9$ and $A .10$ decrease their sensitivity with the input, in fact they are at the denominator in equation (1). This is another interesting outcome for the development of specific equations that can be used to couple different parameters (attributes) belonging to the same domain.

\section{CONCLUSION}

The proposed analysis demonstrated that when product architectures are modelled according to the CDfA framework (i.e., levels, domains, and attributes), the process shall be considered carefully to avoid the use of meaningless attributes. The paper focuses on the study of attributes sensitivity, through the application of a sensitivity analysis method (i.e., OFAT method) which consists of changing one attribute value at time, keeping other fixed. Attributes were collected inside domains using two mathematical operators (i.e., Mean and $R M S$ ) and another equation derived from engineering consideration. Moreover, the analysis was repeated for two different baselines. The obtained results showed that: i) the use of the Mean operator to collect attributes inside a domain allows to obtain constant sensitivity for all attributes regardless the baseline, ii) the use of $R M S$ operator to collect attributes inside a domain gives different sensitivity results according to the baseline, and iii) the use of ad-hoc mathematical models might lead to inconsistent results. Furthermore, by increasing the number of attributes collected within a domain, the sensitivity of each attribute decreases. However, the proposed analysis presents some shortcomings: the OFAT method does not consider attributes interactions, and, if it is used to estimate sensitivity of models with attribute interactions may lead to different results (e.g., equation used to model the system interface domain). In these cases, other SA methods shall be considered (i.e., global approaches). Moreover, the analysis was performed using a one-level model, thus attributes sensitivity when more than one levels are present were not investigated. If more than a single level is considered, it is reasonable to assume that attributes belonging to lower levels will affect the overall CDfA less, since their effect will be flattered by more computation steps. Further investigation will be performed on this aim. Thus, future developments will require the repetition of the analysis using different SA methods and the study of more complex CDfA models (i.e., more than 1 level). Finally, other mathematical models other than Mean operator and RMS may be considered inside the analysis.

\section{REFERENCES}

AlGeddawy, T. \& ElMaraghy, H. 2013, "Reactive design methodology for product family platforms, modularity and parts integration”, CIRP Journal of Manufacturing Science and Technology, vol. 6, no. 1, pp. 34-43. 
Baylis, K., Zhang, G. \& McAdams, D.A. 2018, "Product family platform selection using a Pareto front of maximum commonality and strategic modularity", Research in Engineering Design, vol. 29, no. 4, pp. 547-563.

Bonvoisin, J., Halstenberg, F., Buchert, T. \& Stark, R. 2016, “A systematic literature review on modular product design", Journal of Engineering Design, vol. 27, no. 7, pp. 488-514.

Boothroyd, G., Dewhurst, P. \& Knight, W. A. 2011, "Product design for manufacture and assembly", CRC Press

Bouissiere, F., Cuiller, C., Dereux, P.-., Malchair, C., Favi, C. \& Formentini, G. 2019, "Conceptual design for assembly in aerospace industry: A method to assess manufacturing and assembly aspects of product architectures", Proceedings of the International Conference on Engineering Design, ICED, pp. 2961.

Bullen, G.N. 1999, “Assembly automation and implementation issues”, SAE Technical Papers

Campolongo, F., Cariboni, J. \& Saltelli, A. 2007, “An effective screening design for sensitivity analysis of large models", Environmental Modelling and Software, vol. 22, no. 10, pp. 1509-1518.

Chen, Y., Peng, Q. \& Gu, P. 2018, "Methods and tools for the optimal adaptable design of open-architecture products", International Journal of Advanced Manufacturing Technology, vol. 94, no. 1-4, pp. 991-1008.

Czitrom, V. 1999, “One-factor-at-a-time versus designed experiments”, American Statistician, vol. 53, no. 2, pp. $126-131$.

Favi, C. \& Germani, M. 2012, “A method to optimize assemblability of industrial product in early design phase: From product architecture to assembly sequence", International Journal on Interactive Design and Manufacturing, vol. 6, no. 3, pp. 155-169.

Favi, C., Formentini, G., Bouissiere, F., Cuiller, C., Dereux, P.-. \& Malchair, C. 2020, "Design for Assembly in the Conceptual Development of Aircraft Systems". Proceedings of the International Conference on Design, Simulation, Manufacturing: The Innovation Exchange ADM 2019: Design Tools and Methods in Industrial Engineering pp 268-278.

Favi, C., Germani, M. \& Mandolini, M. 2016, "Design for Manufacturing and Assembly vs. Design to Cost: Toward a Multi-Objective Approach for Decision-making Strategies during Conceptual Design of Complex Products", Procedia CIRP, pp. 275.

Favi, C., Germani, M. \& Mandolini, M. 2018, "Development of complex products and production strategies using a multi-objective conceptual design approach", International Journal of Advanced Manufacturing Technology, vol. 95, no. 1-4, pp. 1281-1291.

Formentini, G., Favi, C., Bouissiere, F., Cuiller, C., Dereux, P.-E., Guillaume, R. and Malchair, C. 2020 "Extrapolation of Design Guidelines During the Conceptual Design Phase: A Method to Support Product Architecture Design,". Proceedings of the Design Society: DESIGN Conference. Cambridge University Press, 1, pp. 857-866.

Hamby, D.M. 1994, "A review of techniques for parameter sensitivity analysis of environmental models", Environmental monitoring and assessment, vol. 32, no. 2, pp. 135-154.

Krishnan, V. \& Ulrich, K.T. 2001, "Product development decisions: A review of the literature", Management Science, vol. 47, no. 1, pp. 1-21.

Marino, S., Hogue, I.B., Ray, C.J. \& Kirschner, D.E. 2008, “A methodology for performing global uncertainty and sensitivity analysis in systems biology", Journal of theoretical biology, vol. 254, no. 1, pp. 178-196.

Pahl, G., Beitz, W., Feldhusen, J. \& Grote, K.-. 2007, "Engineering design: A systematic approach" in Engineering Design: A Systematic Approach, pp. 1-617.

Pianosi, F., Beven, K., Freer, J., Hall, J.W., Rougier, J., Stephenson, D.B. \& Wagener, T. 2016, "Sensitivity analysis of environmental models: A systematic review with practical workflow”, Environmental Modelling and Software, vol. 79, pp. 214-232.

Ravalico, J.K., Maier, H.R., Dandy, G.C., Norton, J.P. \& Croke, B.F.W. 2005, “A comparison of sensitivity analysis techniques for complex models for environmental management", MODSIM05 - International Congress on Modelling and Simulation: Advances and Applications for Management and Decision Making, Proceedings, pp. 2533.

Saltelli, A. \& Annoni, P. 2010, "How to avoid a perfunctory sensitivity analysis", Environmental Modelling and Software, vol. 25, no. 12, pp. 1508-1517.

Selvaraj, P., Radhakrishnan, P. \& Adithan, M. 2009, “An integrated approach to design for manufacturing and assembly based on reduction of product development time and cost", International Journal of Advanced Manufacturing Technology, vol. 42, pp. 13-29.

Stone, R.B., McAdams, D.A. \& Kayyalethekkel, V.J. 2004, “A product architecture-based conceptual DFA technique”, Design Studies, vol. 25, no. 3, pp. 301-325.

Suzuki, T., Ohashi, T., \& Asano, M. 2003, “Assembly reliability evaluation method (AREM)”, CIRP Annals Manufacturing Technology, 52(1), 9-12.

Yu, S., Yang, Q., Tao, J., Tian, X. \& Yin, F. 2011, "Product modular design incorporating life cycle issues - Group Genetic Algorithm (GGA) based method”, Journal of Cleaner Production, vol. 19, no. 9-10, pp. 1016-1032.

Saltelli, A., Ratto, M., Tarantola, S. \& Campolongo, F. 2006, "Sensitivity analysis practices: Strategies for model-based inference”, Reliability Engineering and System Safety, vol. 91, no. 10-11, pp. 1109-1125. 